\title{
BMJ Open Why do patients develop severe pressure ulcers? A retrospective case study
}

\author{
Lisa Pinkney, ${ }^{1}$ Jane Nixon, ${ }^{2}$ Lyn Wilson, ${ }^{2}$ Susanne Coleman, ${ }^{2}$ \\ Elizabeth McGinnis, ${ }^{3}$ Nikki Stubbs, ${ }^{4}$ Carol Dealey, ${ }^{5}$ Andrea Nelson, ${ }^{6}$ \\ Malcolm Patterson, ${ }^{7}$ Justin Keen ${ }^{1}$
}

To cite: Pinkney L, Nixon J, Wilson L, et al. Why do patients develop severe pressure ulcers?

A retrospective case study. BMJ Open 2014;4:e004303. doi:10.1136/bmjopen-2013004303

- Prepublication history for this paper is available online. To view these files please visit the journal online (http://dx.doi.org/10.1136/ bmjopen-2013-004303).

Received 22 October 2013 Revised 29 November 2013 Accepted 4 December 2013

CrossMark

For numbered affiliations see end of article.

Correspondence to Dr Justin Keen;

j.keen@leeds.ac.uk

\section{ABSTRACT}

Objectives: This study focuses on the ways in which the organisational context can influence the development of severe pressure ulcers. Severe pressure ulcers are important indicators of failures in the organisation and delivery of treatment and care. We have a good understanding of patients' risk factors, but a poor understanding of the role played by the organisational context in their development.

Setting: The study was undertaken in six sites in Yorkshire, England. The settings were sampled in order to maximise diversity, and included patients' own homes, acute hospital medical and surgical wards, a community hospital and a nursing home during a period of respite care.

Participants: Data were collected about eight individuals who developed severe pressure ulcers, using a retrospective case study design. The data sources included interviews with individuals with severe pressure ulcers, and with staff who had treated and cared for them, and clinical notes.

Results: 4 accounts indicated that specific actions by clinicians contributed to the development of severe pressure ulcers. Seven of the 8 accounts indicated that they developed in organisational contexts where (1) clinicians failed to listen and respond to the patients' or carers' observations about their risks or the quality of their treatment and care, (2) clinicians failed to recognise and respond to clear signs that a patient had a pressure ulcer or was at risk of developing one and (3) services were not effectively coordinated.

Conclusions: Patient accounts could only be partially explained in terms of specific events or sequences of events. The findings support the conclusion that there was general acceptance of suboptimal clinical practices in 7 of the 8 contexts where patients developed severe pressure ulcers.

\section{INTRODUCTION}

The European Pressure Ulcer Advisory Panel/National Pressure Ulcer Advisory Panel (EPUAP/NPUAP) defines a pressure ulcer as, 'localised injury to the skin and/or underlying tissue usually over a bony

\section{Strengths and limitations of this study}

- This study contributes to our understanding of a poorly understood process, the development of a severe pressure ulcer.

- Few previous studies have explicitly sought to discriminate between psychological and broader organisational explanations for adverse events in healthcare settings.

- The diversity of patients who develop severe pressure ulcers, and of the settings where they occur, raises a risk of sampling bias.

- The retrospective study design brings with it a risk of hindsight bias.

prominence, as a result of pressure, or pressure in combination with shear'. 'Pressure ulcers are a significant source of pain and distress for the individuals who develop them. ${ }^{2}$ In recent years, the importance of severe pressure ulcers as indicators of poor quality and safety of health services has been recognised. Category 2 ulcers or above, as rated on the EPUAP/NPUAP 1-4 scale, are classed as reportable incidents in official guidelines in the National Health Service (NHS) in England. ${ }^{3}$ Category 3 and 4 ulcers (which involve injury deep into the skin, muscle or bone) are widely termed severe pressure ulcers, and have to be reported as serious untoward incidents. ${ }^{4}$ Pressure ulcers are also one of the 4 patient safety indicators in a new NHS monitoring tool. ${ }^{5}$

There are two distinct ways of thinking about patients' risks of developing pressure ulcers. The first is based on the assumption that all PU risks are associated with patients' health status or their behaviour. The implication is that clinicians should focus on identifying patients who are at risk, assess the nature and scale of their risks and design clinical interventions to reduce them. We have a good understanding of patients' risk 
factors. ${ }^{6}$ The second way of thinking starts from a different assumption, which is that the quality of treatment and care can also influence patients' risks of developing pressure ulcers. Patients who are at risk are more likely to develop them in settings where quality of care is poor. The events at Mid Staffordshire NHS Foundation Trust, where at one point dozens of pressure ulcers (PUs) were being reported every month, help to underline the significance of this point. ${ }^{7}$

We currently have a relatively poor understanding of the ways in which the wider organisational context contributes to their prevention or development. A small number of studies have indicated that it plays a role, but the nature and significance of that role remain to be elucidated. ${ }^{8}$ This study focuses on the ways in which the organisational context can influence the development of severe pressure ulcers. It focuses on identifying the best explanation for their development, using explanations derived from the patient safety literature, which advances psychological and sociological explanations for errors and adverse events. ${ }^{9}$

\section{METHODS}

Severe pressure ulcers occur relatively rarely, and can develop in a wide range of settings, and it is currently not possible to predict who will develop them and who will not. ${ }^{6}$ It was not therefore practical to study their development prospectively. If, for example, we had prospectively identified patients with category 2 ulcers, in order to evaluate differences between those who developed a category 3 or 4 ulcer and those who did not, our presence would have drawn attention to the significance of the pressure ulcers. It is likely to have prompted swift action by the local clinical team, and it seems reasonable to predict that few, or even none, of the category 2 pressure ulcers would have progressed to category 3 or 4 . As a result, we would have biased our observations, possibly substantially, and could not have been confident that we had observed the whole development process, from the earliest signs and symptoms to the point where action was taken. It was, though, possible to reconstruct the events that lead to the development of severe pressure ulcers retrospectively. We undertook a retrospective case study, where severe pressure ulcers were end points and also indicators of adverse outcomes of treatment and care. A process tracing case study method was used, focusing on the experiences of eight individuals in Yorkshire, England. ${ }^{10}$ Each account took, on an average, 4 months to create, from the initial interview with an individual to the signing off of a detailed account of the development of that individuals' severe pressure ulcer.

\section{Primary data collection}

Research ethics committee approval and local research governance approvals from six study sites were obtained. Participants were sampled purposively in order to maximise the diversity of individuals and the contexts in which they developed severe pressure ulcers. The settings included patients' own homes, acute hospital medical and surgical wards, a community hospital and a nursing home during a period of respite care. Sampling was also pragmatic: individuals who had developed a category 3 or 4 pressure ulcer were identified by members of the local tissue viability nurse teams. Consent to participate was obtained from patients, and where appropriate also from their main carers.

Data were collected by a field researcher with a nonclinical background from five sources, namely interviews with individuals who had developed a severe pressure ulcer (and where relevant also their main carers), interviews with clinical and other staff who had been involved in their care, clinical records, other documents relevant to the account such as critical incident reports and relevant local policy documents, for example, on assessment of risks of skin breakdown (figure 1, stage 1). Interviews were open-ended and in-depth, and are listed in table 1. A total of 70 interviews were conducted across the eight accounts. The site principal investigator, who in each case was a nurse with a specialist interest in tissue viability, collated patient notes in a parallel exercise, following current practice in the NHS in England for root cause analyses.

\section{Development of retrospective accounts}

The initial accounts each had two components. The first consisted of verbatim passages of the patient/carer interview, which captured their explanations of the events that led to their severe pressure ulcers. Second, a Microsoft Access database was created for each account,

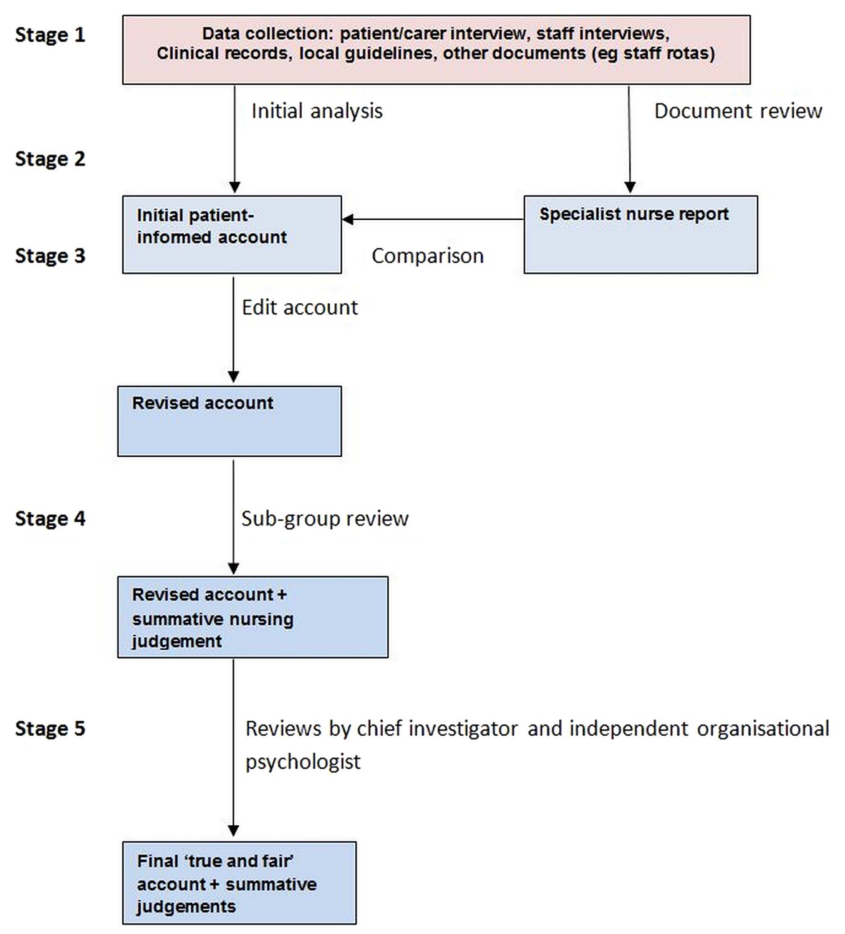

Figure 1 Analysis and review of individual accounts. 


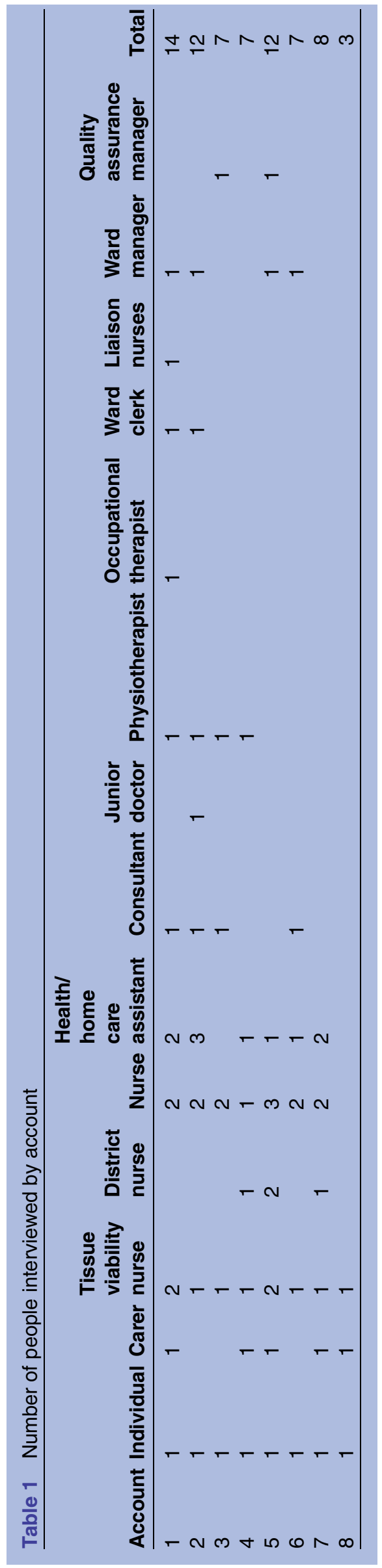

and used to organise decisions and actions into a chronological sequence, with patient and carer data in one column, other interview data in the second column and records and other documentary sources in the third column (see figure 1, stage 2). The presentation of data in parallel columns made it possible to identify consistencies and inconsistencies between different data sources, and also the 'strength' of evidence available about each event, reflected in the number and quality of sources. Data from the two components were used to identify a provisional timeline of events for each account.

A tissue viability nurse specialist from the relevant study site undertook a parallel review, based solely on available patient records and on other available documentation, including local guidelines and critical incident reports (ie, not including the patient/carer interview). The method followed the guidance for reviews of critical incidents in the NHS in England. The nurse wrote a report, identifying key decisions and actions in chronological order, including departures from local guidelines. The field researcher and tissue viability nurse specialist then met and compared their accounts, identifying consistencies and inconsistencies, for example, actions that the nurse judged as important, which were not included in the initial patient-driven account. Timelines were revised in the light of additional facts or insights generated (stage 3).

\section{Refinement of the accounts}

The subsequent stages of the analysis were designed to minimise some of the risks of bias known to be associated with retrospective analysis, notably hindsight bias, through review of each account by researchers with different backgrounds. The initial summaries of each account were reviewed by a subgroup of nursing members of the research team: one independent hospital-based and one independent community-based tissue viability nurse specialist and one of the co-chief investigators (stage 4).

The accounts were analysed in two ways. First, they were used to identify any errors-in the opinion of the subgroup-made in the decisions and actions recorded in each account. The specialist nurse reports, in particular, were important to help identify decisions made and actions taken, and hence provided an evidential basis for identifying errors of omission or commission. Each point was checked by going back to primary data sources. This produced an account that could be deemed to be 'true and fair'. On the basis of the account, the clinical subgroup made expert judgements about departures from the treatment and care that each individual might reasonably have expected to receive. These departures-such as failures to undertake proper risk assessments or to act when there were clear signs of skin redness or a category 1 ulcer-were possible precipitating, or contributing, events in the development of each severe pressure ulcer. Second, drawing on Yin's 
strategy for discriminating between hypotheses in case studies, ${ }^{11}$ clinical subgroups were asked to select one or more of five explanations for the events portrayed in an account. The five explanations were that a severe pressure ulcer

1. Could not have been avoided;

2. Developed following an isolated mistake made by a clinician;

3. Developed following a sequence of unconnected errors;

4. The organisational context made development more likely;

5. Developed for another reason, not covered by the first four.

The first explanation captures a situation where clinical staff did everything that might reasonably have been expected. The second reflects the dominant assumption in the patient safety literature, and is supported by some evidence about pressure ulcer development. ${ }^{12}{ }^{13}$ The third is a version of Reason's 'Swiss Cheese' model, and again has some support in the pressure ulcer literature. ${ }^{14-17}$ The fourth explanation, which also has some support in the pressure ulcer literature, focuses on the role of the organisational context, highlighted in the institute of medicine's report, To Err Is Human. ${ }^{18-21}$ The implicit assumption underpinning this explanation is that suboptimal treatment and care are provided, compared with the overall treatment and care that an individual might reasonably expect to receive, as judged by the clinical subgroup and subsequent reviewers. The fifth explanation is a logical extension to the first four explanations, retaining the possibility of a novel explanation.

The revised accounts and explanations were reviewed by the non-clinical co-chief investigator and then by an organisational psychologist who had not been involved in the earlier stages (stage 5). The reviews focused on the coherence of each account, that is, the extent to which the patient's explanation and/or the nurses' judgements made sense of the available evidence. In the final step in the analysis, the eight accounts were analysed inductively in order to identify themes that were common across the accounts. ${ }^{22}$

\section{RESULTS}

The study demonstrates that it is possible to develop detailed retrospective accounts of events, and to use them to judge which of five possible explanations best fits the available evidence. The large volumes of data collected and included in the timeline appear to have minimised problems that might have arisen as a result of 'missing data'. The iterative review process, involving reviewers with different backgrounds, appears to have minimised the risks of misinterpretation. As we note in the Discussion section, though, the results may still be subject to a number of biases.

The eight individuals were selected, in part, to maximise diversity (see table 2). There were, therefore, marked differences in their personal characteristics and in their treatment and care. They were all, though, at high risk of developing pressure ulcers, or of existing pressure ulcers deteriorating. Different explanations were offered by those interviewed for the development of severe pressure ulcers. For example, in a number of accounts, some staff interviewed blamed patients, on the basis that they had not complied with advice on managing their risks, for example, shifting position regularly. But patients themselves, in the same accounts, pointed to specific actions or omissions-failure to be turned regularly overnight, to provide a specialised mattress or to respond to patients' comments about their own risks.

\section{Elimination of hypotheses}

The diverse group of individuals all had the same outcome, a severe pressure ulcer. In one account (\#8), development was judged to be unavoidable, because the individual concerned developed a severe pressure ulcer in her own home, before any health professional saw her. The other seven accounts were deemed to involve avoidable severe pressure ulcers in the specialist nurse

Table 2 Individuals and settings

\begin{tabular}{|c|c|c|}
\hline Account & Individual & Setting \\
\hline 1 & 38-year-old woman with paraplegia & Acute hospital, surgical ward \\
\hline 2 & $\begin{array}{l}65 \text {-year-old woman with long-term chronic neurological condition and } \\
\text { undiagnosed infection }\end{array}$ & Acute hospital, medical ward \\
\hline 3 & 75-year-old man with multiple chronic health problems and acute infection & $\begin{array}{l}\text { Community hospital, rehabilitation } \\
\text { ward }\end{array}$ \\
\hline 4 & $\begin{array}{l}\text { 37-year-old woman with long-term degenerative congenital neurological } \\
\text { condition }\end{array}$ & At home \\
\hline 5 & $\begin{array}{l}\text { 90-year-old man with multiple chronic health problems and undiagnosed } \\
\text { acute illness }\end{array}$ & Acute hospital, surgical ward \\
\hline 6 & $\begin{array}{l}\text { 39-year-old woman in hospital for acute undiagnosed postoperative surgical } \\
\text { complications }\end{array}$ & Acute hospital, surgical ward \\
\hline 7 & 65 -year-old man with quadriplegia & $\begin{array}{l}\text { At home, respite care and acute } \\
\text { hospital }\end{array}$ \\
\hline 8 & 89-year-old woman who fell at home & At home \\
\hline
\end{tabular}


Table 3 Summative judgements by account

\begin{tabular}{|c|c|c|c|c|c|}
\hline Account & Unavoidable & Single/isolated event & Sequence of events & $\begin{array}{l}\text { Environment made } \\
\text { development more likely }\end{array}$ & Other explanation \\
\hline 1 & & & & $\bullet$ & \\
\hline 2 & & & $\bullet$ & - & \\
\hline 3 & & $\bullet$ & & $\bullet$ & \\
\hline 4 & & & $\bullet$ & $\bullet$ & \\
\hline 5 & & & & $\bullet$ & \\
\hline 6 & & & $\bullet$ & $\bullet$ & \\
\hline 7 & & & & $\bullet$ & \\
\hline 8 & $\bullet$ & & & & \\
\hline
\end{tabular}

reports and the reviews by the clinical subgroup, on the basis that there was clear evidence of departures from the care that the patient might reasonably have expected to receive. The second and third hypotheses were causal in nature: in one account (\#3), there was a single precipitating event, and there was a sequence of precipitating events in three others (\#2, \#4 and \#6). In each of the four cases, though, reviewers judged that, while specific events played a role, they provided only part of the explanation. In these cases, and in the three remaining ones-7 of the 8-the clinical subgroup and subsequent reviewers judged that the organisational context made development of a severe pressure ulcer more likely, compared with the overall treatment and care that the individual might reasonably have expected to receive (see table 3). None of the eight accounts, in the view of the clinical subgroup or subsequent reviewers, supported an alternative explanation.

\section{The organisational context}

The next step was to understand how the organisational context contributed to the development of severe pressure ulcers. Inductive analysis of the eight accounts led to the identification of three main themes. First, the 'voices' of the individuals who developed severe pressure ulcers were not heard by staff. As noted above, the individuals themselves behaved differently and had different relationships with clinical staff, but failures to heed information were evident in several accounts. For example, there were examples of patients making repeated appeals for pain and discomfort to be addressed, and expressing concerns about their own well-being, which were not heeded over periods of hours or even days. In some instances, these appeals seem to have been dismissed by staff, that is, they were heard but not taken seriously. Patients were also blamed for the development of their pressure ulcers, on the basis that they did not comply with instructions they were given, and branded as 'difficult'-even when they had cognitive impairments.

Second, there were failures to recognise and act on warning signs. Risk assessments were not undertaken when they should have been, in some cases only being undertaken several days after admission to an acute hospital ward. Evidence of pre-existing clinical risks in records was not acted on in 6 of the 7 patients where the environment was judged to have contributed to development. Action was not taken promptly when overt evidence-including the presence of a category 2 pressure ulcer-was identified. Conversely, there was evidence of poor documentation, so that adherence with patients' care plans was not recorded, and, in some instances, direct evidence of skin redness or a pressure ulcer was not recorded. Some healthcare assistants, who provided direct care, observed that they lacked the appropriate training to identify and record risks, or were not allowed to record them.

Third, there were coordination failures, between patients, carers and staff, staff in the same setting, between staff in different settings in the same organisation (eg, two wards) and between staff in different organisations. Sometimes this was manifested as interprofessional communication failure, and in some cases there was poor communication between the same professional groups in two locations. One example of the latter came in a postoperative setting, where risks were not properly communicated between the anaesthetic recovery unit and the postoperative ward. In other accounts, records were not moved with an individual, so that key information was not available in a new setting. It would be possible to interpret these points as clear evidence of failures by individuals or teams. There is a corollary to this point: nurses and healthcare assistants, in particular, could find themselves working in conditions where they had limited information about individuals and their risks, for example, where patients had unknown diagnosis, or where records had not travelled with the patient from another location. It is possible, therefore, that individual members of staff behaved reasonably in the contexts in which they found themselves. The problems observed could be attributed to weaknesses in the overall coordination of treatment and care.

\section{DISCUSSION}

This study sought to explain why patients develop severe pressure ulcers, by reconstructing events retrospectively, and then discriminating between alternative explanations for their development. The principal explanation 
is that severe pressure ulcers developed in organisational contexts where there were failures in the overall governance of services. Specifically, they were characterised by one or more of (1) clinicians failing to listen to the patients' or carers' observations about their risks or the quality of their treatment and care, (2) clinicians failing to recognise and respond to clear signs that a patient had a pressure ulcer or was at risk of developing one, and (3) services not being effectively coordinated. In four of the accounts it was possible to identify specific, or causal, precipitating events, but in each case these events occurred in organisational contexts where there were more general governance problems.

As noted in the Methods section, the study was designed in significant part in order to minimise biases in the data collection and analysis in a retrospective, observational study. This study suggests that a novel method, based on tracing back the course of events retrospectively from a known outcome, can be used to reconstruct key events. The resulting accounts can be subjected to detailed review, and used to discriminate between alternative explanations for those events, and in the process preserve the 'voices' of the individuals affected. This said, it is important to stress that there are a number of sources of bias, starting with selection bias: while the sampling strategy maximised diversity, the eight accounts are of individuals who were willing and able to consent to participate. The initial presentation of the timelines and the backgrounds of the analysts and reviewers are also potential sources of bias. A study team with different clinical or disciplinary backgrounds might have arrived at different judgements: for example, a team with backgrounds in human factors psychology might have placed greater weight on single events or sequences of events. There is also a risk, using a retrospective design, of hindsight bias, particularly in reviewers assuming that staff must have known more than they actually did, and should therefore have acted differently. ${ }^{23}$ The sequential and iterative review process has, we hope, served to minimise these biases, but we cannot say that they have been eliminated.

We can interpret our findings in the context of the patient safety literature. Reason ${ }^{17}$ points out that investigations of accidents, across many industries, have changed significantly over the past 50 years. An early focus on equipment failure gave way, in the 1970s and 1980s, to focus on human error, and then more recently to accounts that focused on systems and cultural issues. In spite of this, many current patient safety studies focus on causal explanations, based either on patient characteristics or errors made by individual clinicians. These were represented by the second and third explanations. Relatively few studies focus on the wider organisational context represented by the fourth explanation. ${ }^{11}$ The findings reported here only partially support the second or third explanation. Only one patient was deemed to have an unavoidable severe pressure ulcer-because service providers were unaware of a fall at home- supporting the first explanation, and there was no support for a fifth, alternative, explanation. The overall findings are, though, consistent with explanations that emphasise systems and culture.

In the literature on the role of the organisational context on patient safety, explanations tend to emphasise either systems or culture. The findings suggest that, for people who developed severe pressure ulcers, both were important. In relation to systems-based explanations, the evidence about the poor coordination of services is broadly consistent with the arguments in To Err Is Human, namely that many safety failures are essentially system failures. ${ }^{21}$ Drawing on the work of Perrow ${ }^{23}$ and others, the institute argued that accidents are more likely in systems that are inherently complex-having many interconnected elements. The findings in this study supported the observation that there were coordination failures among services that were loosely coupled with one another, that is, generally run independently of one another, but needing to coordinate with one another. For example, there were communication failures between wards at times when there were major ward reorganisations, so that key information was not passed on. Similarly, one of the community-based accounts revealed that the individual was in receipt of a hospital service that community staff were unaware of, and hence could not take into account in risk assessment or care planning.

At the same time, the failures to listen properly to patients-and even dismiss their concerns-and to act when a superficial pressure ulcer was present emphasise the importance of prevailing cultural norms. The evidence suggests that the environments where severe pressure ulcers developed were ones where staff were under time pressure, where there were problematic relationships between staff groups, and where staff were defensive and prepared to attribute failures to colleagues or to the 'difficult' behaviour of patients. This takes us away from a causal explanation, linking clinical actions to the development of severe pressure ulcers, to one where the explanation is that prevailing norms substantially influenced the decisions and actions of individuals, which in turn led to the errors of commission and omission described above. Clinicians adopted risky work routines that were not appropriate for the vulnerable patients who were in their care. Severe pressure ulcers developed in contexts where there was normalisation of deviance, a phenomenon where risky practices become the norm in a work setting, and staff either do not recognise the extent of the risks they are taking or are aware of them but underestimate them. ${ }^{24}$ This resonates with wider concerns about the culture in parts of the NHS in England, where staff can be defensive and quick to blame others, rather than being open and prepared to learn from adverse events. ${ }^{6}$

Author affiliations

${ }^{1}$ Leeds Institute of Health Sciences, University of Leeds, Leeds, UK

${ }^{2}$ Leeds Institute of Clinical Trials Research, University of Leeds, Leeds, UK 
${ }^{3}$ Department of Tissue Viability, Leeds Teaching Hospitals NHS Trust, Leeds, UK

${ }^{4}$ Tissue Viability Service, Leeds Community Healthcare NHS Trust, Leeds, UK ${ }^{5}$ College of Medical and Dental Sciences, University of Birmingham, Birmingham, UK

${ }^{6}$ School of Healthcare, University of Leeds, Leeds, UK

${ }^{7}$ Management School, University of Sheffield, Sheffield, UK

Contributors JN and JK conceived and designed the study initially. The study design was developed iteratively over a period of months by CD, JK, EM, JN, LP and NS. LP undertook primary data collection. Additional data were collected and collated by EM, NS and LW. JK drafted the article and revised it critically on the basis of comments from the other authors. All authors were formally involved in the analysis and interpretation of the findings. Coauthors will give final approval of the version to be published.

Funding This article presents independent research funded by the National Institute for Health Research (NIHR) under its Programme Grants for Applied Research Programme (RP-PG-0407-10056).

Competing interests None.

Ethics approval Leeds West Ethics Committee.

Provenance and peer review Not commissioned; externally peer reviewed.

Data sharing statement No additional data are available.

Open Access This is an Open Access article distributed in accordance with the Creative Commons Attribution Non Commercial (CC BY-NC 3.0) license, which permits others to distribute, remix, adapt, build upon this work noncommercially, and license their derivative works on different terms, provided the original work is properly cited and the use is non-commercial. See: http:// creativecommons.org/licenses/by-nc/3.0/

\section{REFERENCES}

1. National Pressure Ulcer Advisory Panel/European Pressure Ulcer Advisory Panel. Pressure ulcer treatment. NPUAP/EPUAP, 2009.

2. Gorecki C, Brown JM, Nelson EA, et al. Impact of pressure ulcers on quality of life in older patients: a systematic review. J Am Geriatr Soc 2009;57:1175-83

3. National Institute for Clinical Excellence. Pressure ulcersprevention and treatment. London: NICE, 2005.

4. National Patient Safety Agency. National framework for reporting and learning from serious incidents requiring investigation. London: NPSA, 2010.

5. http://www.ic.nhs.uk/thermometer (accessed 07/05/2013).
6. Coleman S, Gorecki C, Nelson EA, et al. Patient risk factors for pressure ulcer development: systematic review. Int J Nurs Stud 2013;50:974-1003.

7. Francis $\mathrm{R}$ (Chair). Independent Inquiry into care provided by Mid-Staffordshire NHS Foundation Trust, January 2005-March 2009. London: TSO, 2013.

8. Berlowitz DR, Young GJ, Hickey EC, et al. Quality improvement implementation in the nursing home. Health Serv Res 2003; 38(1 Part 1):65-83.

9. Waring J, Rowley E, Dingwall R, et al. A Narrative Review of the UK's Patient Safety Research Portfolio. J Health Serv Res Policy 2010;15:26-32.

10. George A, Bennett A. Case studies and theory development in the social sciences. Cambridge, MA: MIT Press, 2005.

11. Yin R. Case study research: design and methods. London: Sage, 2008.

12. van Gaal B, Schoonhoven L, Hulscher M, et al. The design of the SAFE or SORRY? study: a cluster randomised trial on the development and testing of an evidence based inpatient safety program for the prevention of adverse events. BMC Health Serv Res 2009;9:58-65.

13. van Gaal B, Schoonhoven L, Vloet $L$, et al. The effect of the SAFE or SORRY? programme on patient safety knowledge of nurses in hospitals and nursing homes: a cluster randomised trial. Int $J$ Nurs Stud 2010;47:1117-25.

14. Chaves L, Grypdonck M, Defloor T. Pressure ulcer prevention in homecare: do Dutch homecare agencies have an evidence-based pressure ulcer protocol? J Wound Ostomy Continence Nurs 2006;33:273-80.

15. Dopierala L, Szewczyk M, Cierzniakowska K, et al. Level of preparation for preventive procedures and pressure ulcer treatment in health care units from the Kujawsko-Pomorski region. Adv Med Sci 2007:52(Suppl 1):81-4.

16. Schubert M, Clarke S, Glass T, et al. Identifying thresholds for relationships between impacts of rationing of nursing care and nurse- and patient-reported outcomes in Swiss hospitals: a correlational study. Int J Nurs Stud 2009;46:884-93.

17. Reason J. The human condition. Farnham: Ashgate, 2008:131-9.

18. Castle NG. Administrator turnover and quality of care in nursing homes. Gerontologist 2001;41:757-67.

19. Kennedy M. Improving pressure ulcer prevention in a nursing home: action research. Br J Community Nurs 2005;10:S6-16.

20. Yang KP. Relationships between nurse staffing and patient outcomes. J Nurs Res 2003;11:149-58.

21. Institute of Medicine. To Err Is Human. Washington, DC: IoM, 1999.

22. Pawson R. Middle range realism. Eur J Sociol 2000;41:283-325.

23. Perrow C. Normal accidents. New Edition. Princeton: Princeton University Press, 1999.

24. Vaughan D. The challenger launch decision. Chicago: University of Chicago Press, 1996. 